\title{
Towards Phase Field Models for the Kinetics of Change During Mantle Melting
}

\author{
Christopher J. Grose ${ }^{1,2}$ AND PAUl D. ASIMOW ${ }^{1}$ \\ ${ }^{1}$ Division of Geological and Planetary Sciences, California \\ Institute of Technology, Pasadena, CA 91125, USA. \\ ${ }^{2}$ chrgrose@mail.usf.edu
}

The generation and evolution of mantle melts is a process of fundamental importance to our understanding of the dynamics and history of the Earth. Because melting is a complicated process coupling mechanics, chemical thermodynamics, and heat transport, much recent work has been focused on the formulation of more physically comprehensive models which incorporate the necessary physics and sub-solidus thermodynamic models. However, one aspect of melting which has not received significant attention is the role of kinetics. The chemical differentiation aspect of melting is governed by the diffusion and reaction processes which transport mass among solids and liquids during phase change. Although disequilibrium is thought to be the rule on large scales to maintain compositional mantle heterogeneities, there is some small scale at which equilibrium applies over the time-scales of melting and intermediate scales where the kinetics of change determine the evolution and interactions within the melting system. Because the scales at which kinetics is relevant could be as small as the grain size, implications for the chemical evolution of melts and their residues and resulting consequences for the evolution of the mantle and lithosphere could be important.

To understand the role of kinetics during mantle evolution processes such as melting we are developing a new computational framework for grain-scale non-equilibrium thermodynamics using the phase field method. In this approach, evolution equations for interfacial dynamics, chemical transport, and nucleation are coupled with thermodynamic and kinetic databases. Phase field methods can be used to self-consistently model the underlying phenomena of melting minerals and rocks. We will describe the basic features of these models, challenges in their development and interpretation, and our attempt to apply it to the problem of decompression melting of the mantle. 